\title{
Improving the use of benzodiazepines-Is it possible? A non-systematic review of interventions tried in the last 20 years
}

\author{
Alesha J Smith*, Susan E Tett
}

\begin{abstract}
Background: Benzodiazepines are often used on a long term basis in the elderly to treat various psychological disorders including sleep disorders, some neurological disorders and anxiety. This is despite the risk of dependence, cognitive impairment, and falls and fractures. Guidelines, campaigns and prescribing restrictions have been used to raise awareness of potentially inappropriate use, however long term use of benzodiazepine and related compounds is currently increasing in Australia and worldwide. The objective of this paper is to explore interventions aimed at improving the prescribing and use of benzodiazepines in the last 20 years.

Methods: Medline, EMBASE, PsychINFO, IPA were searched for the period 1987 to June 2007.

Results: Thirty-two articles met the study eligibility criteria (interventions solely focusing on increasing appropriate prescribing and reducing long term use of benzodiazepines) and were appraised. Insufficient data were presented in these studies for systematic data aggregation and synthesis, hence critical appraisal was used to tabulate the studies and draw empirical conclusions. Three major intervention approaches were identified; education, audit and feedback, and alerts.

Conclusions: Studies which used a multi-faceted approach had the largest and most sustained reductions in benzodiazepines use. It appears that support groups for patients, non-voluntary recruitment of GPs, and oral delivery of alerts or feedback may all improve the outcomes of interventions. The choice of outcome measures, delivery style of educational messages, and requests by GPs to stop benzodiazepines, either in a letter or face to face, showed no differences on the success rates of the intervention.
\end{abstract}

\section{Background}

Benzodiazepines are used to treat various psychological disorders including sleep disorders, some neurological disorders and aspects of addiction and anxiety [1]. Utilization patterns show variation in the use of benzodiazepines over the last 2 decades [2,3]. During the 1990's there was much publicity around the harmful effects of long-term use of benzodiazepines in Australia, including new guidelines and efforts to increase community awareness [4-6]. This resulted in a decrease in prescribing of benzodiazepines, however since the end of these campaigns, use has been continually rising, with a $21 \%$ increase in utilisation by concession beneficiaries in

\footnotetext{
* Correspondence: alesha.smith@uq.edu.au
University of Queensland, School of Pharmacy, Brisbane, Queensland, 4072,

* Correspondence: alesha.smith@uq.edu.au
University of Queensland, School of Pharmacy, Brisbane, Queensland, 4072, Australia
}

(c) 2010 Smith and Tett; licensee BioMed Central Ltd. This is an Open Access article distributed under the terms of the Creative Commons Attribution License (http://creativecommons.org/licenses/by/2.0), which permits unrestricted use, distribution, and reproduction in any medium, provided the original work is properly cited.
Australia (elderly, over 65 years of age, and those with a low income or receiving benefits) from 2000 to 2006 [7].

It is known that prescribers and healthcare consumers need to be regularly reminded about reviewing longterm/chronic treatments and what the current best practice guidelines are $[8,9]$. This is especially relevant to benzodiazepines, as Australian guidelines state that benzodiazepines should be reserved for short term use only, be part of a broader treatment plan, and be prescribed at minimum doses $[10,11]$. This is due to the risks, including dependence, cognitive impairment, and falls and fractures [12-15]. Patients over the age of 65 years are particularly susceptible to these side effects due to accumulation and existing frailty $[16,17]$.

Research has shown that these restrictions are not being implemented as the use of benzodiazepines continues to rise in Australia [7]. Whilst there have been a 
number of reviews exploring interventions to improve prescribing or change prescribing behavior $[18,19]$, none have focused specifically on issues and interventions surrounding benzodiazepine use. It is therefore timely that a fresh approach is taken improve this problem.

The objective of this paper was to evaluate interventions aimed at changing the use of benzodiazepines over the last 20 years. This time period was chosen capture the interventions implemented during the $1980 \mathrm{~s}$ and $1990 \mathrm{~s}$ when there was increased focus on improving benzodiazepine use as well as the recent introduction of the newer benzodiazepine-related drugs ( $\mathrm{z}$-drugs). This evaluation will assist in determining a future effective strategy designed to increase the appropriateness of prescribing and use of benzodiazepine and related compounds.

\section{Methods \\ Study Eligibility Criteria}

Studies were included if they met the inclusion criteria, those which aimed to change benzodiazepine prescribing in any population either through education, using outreach or by raising awareness with consumers and/or health professionals, all study designs and locations were included for the period 1987-June 2007.

\section{Information Sources}

A search was made of the following databases, Medline (via Ovid), EMBASE, PsychINFO and International Pharmaceutical Abstracts using the keywords (benzodiazepine(s) and anxi*) alone and in combination with intervention strategy(ies), educat" campaign(s), health intervention, intervention and utilis(z)ation. The search was limited to the English language. The reference lists of the retrieved articles and reviews were used to search for additional articles. A citation search was conducted and grey literature was also reviewed. The last search was completed in June 2007.

\section{Study Selection}

The retrieved articles were screened by title (AS) any which clearly did not include an intervention about improving prescribing or use of benzodiazepines were excluded. Then abstracts for the remaining articles were scanned (AS) those studies which met the inclusion criteria were retrieved after abstract examination, for full text analysis.

Exclusion upon full text analysis was pre-determined for the following types of studies:

(a) studies only comparing 2 or more different dosage regimens or different ways of managing withdrawal (i.e. after the actual decision to change use had occurred). (b) studies that did not distinctly report on the rates of prescribing or use of benzodiazepines (i.e. studies that intervened in overall psychotropic prescribing without differentiating benzodiazepines; or those not reporting rates of prescribing or use).

(c) studies that conducted an intervention in specific disease populations for whom benzodiazepines may be clinically indicated for continuous use (i.e. people with schizophrenia, epilepsy, or opioid drug users).

(d) reviews, double publications, animal studies and letters.

Any articles for which inclusion was uncertain were assessed by a second author (ST) until consensus was reached. All included studies were critically appraised and categorized into 1 of 3 groups; educational interventions, audit and feedback interventions, and alert interventions.

\section{Summary measures}

For the purposes of this manuscript, an improvement in the use of benzodiazepines was defined by each study's pre-determined criteria. The results reported are in the same format as they were presented in the original article, this includes results presented in Table 1, 2 and 3, e.g. more appropriate use may be defined by the authors as a decrease in prescribing, perhaps, or a swap to short-acting benzodiazepine use rather than long acting in the elderly.

\section{Synthesis of results}

Using the above summary measures, it is difficult to quantify 'success' or to quantitatively compare improvement between the studies due to the large variation in design and measurement. One of the major barriers is the definition of appropriateness of use for benzodiazepines used by each different study, and therefore what is meant by an improvement of use. This has been defined in a number of ways; some studies used criteria, such as Beers' Criteria [20], others just using quantities of benzodiazepines to ascertain whether recipients of benzodiazepines should have been receiving these medicines. Beers' Criteria and similar indicators are developed from populations, and it is difficult to apply these in a overarching fashion, without allowing for individual clinical need, so determining 'appropriate/inappropriate' based on these rigid criteria is often controversial [21,22]. Other studies simply indicated that an 'improvement' was a decrease in overall use of benzodiazepines [23]. Due to the variation in reporting and the lack of statistical analysis in many studies, this review has not included a meta analysis, instead the studies have been tabled so that the general attributes and the design of the studies can be compared, critiqued and discussed. 
Table 1 Educational Interventions to decrease benzodiazepine use

\begin{tabular}{|c|c|c|c|c|c|}
\hline Study & Location & $\begin{array}{l}\text { Intervention design: } \\
\text { Educational Letter Interventions Targeting Consumers }\end{array}$ & $\begin{array}{l}\text { Study Design and } \\
\text { Size }\end{array}$ & Result & Follow up \\
\hline $\begin{array}{l}\text { Bashir et al, } \\
1994 \text { [57] }\end{array}$ & London & $\begin{array}{l}\text { Letters sent long term users (>1 year) with a request to } \\
\text { visit their GP and allocated to: } \\
1=\text { informed about the correct use of BZ + self help book. } \\
2=\text { control (no advice) }\end{array}$ & $\begin{array}{l}\text { RCT } \\
11 \text { GP practices } 109 \\
\text { patients. }\end{array}$ & $\begin{array}{l}\text { Sig increase in the number of patients' that reduced BZ } \\
\text { prescriptions in intervention group (18\%) compared to } \\
\text { control (5\%). }\end{array}$ & $\begin{array}{l}6 \text { months post } \\
\text { intervention }\end{array}$ \\
\hline $\begin{array}{l}\text { Towle and } \\
\text { Adams, } 2006 \\
\text { [25] }\end{array}$ & Scotland & $\begin{array}{l}\text { Letter sent to repeat BZ users by pharmacist to tell them } \\
\text { that a step down program had been initiated and invited } \\
\text { them to see their GP for a medication review + repeat BZ } \\
\text { prescriptions were inactivated. Posters displayed in GP } \\
\text { practice. }\end{array}$ & $\begin{array}{l}\text { Convenience Sampling } \\
1 \text { practice, } \\
206 \text { patients. }\end{array}$ & $\begin{array}{l}73 \% \text { collected their step down program. Decreased number } \\
\text { of tablets prescribed by } 64 \% \text {. Only } 23 \% \text { of } 369 \text { patients } \\
\text { stayed on a repeat script. (No statistical analysis). }\end{array}$ & $\begin{array}{l}3 \text { years (end } \\
\text { of the study) }\end{array}$ \\
\hline $\begin{array}{l}\text { Heather } \\
\text { et al, } 2004 \text { [26] }\end{array}$ & UK & $\begin{array}{l}\text { Long term BZ users (> } 6 \text { months) were allocated to: } \\
1=\text { letter from GP asking them to consider cutting down } \\
\text { their BZs. } 2=\text { letter inviting participants to a consultation + } \\
\text { leaflet and self help sleep book. } 3=\text { control. }\end{array}$ & $\begin{array}{l}\text { RCT } \\
7 \text { Practices, } 299 \\
\text { patients. }\end{array}$ & $\begin{array}{l}\text { Sig reduction in letter group (24\%) vs. control (16\%) and } \\
\text { sig reduction in consult ( } 22 \%) \text { vs. control (16\%). No sig } \\
\text { difference between consultation and letter group for BZ } \\
\text { decrease. }\end{array}$ & $\begin{array}{l}6 \text { months post } \\
\text { intervention }\end{array}$ \\
\hline $\begin{array}{l}\text { Cormack et al, } \\
1989[58]\end{array}$ & UK & $\begin{array}{l}\text { Long term BZ users (> } 1 \text { year) were allocated to: } 1=\text { letter } \\
\text { from GP advising patients to cut down BZ use. } 2= \\
\text { invitation to see the GP about cutting down BZ use. } \\
3=\text { control. }\end{array}$ & $\begin{array}{l}\text { CT } \\
5 \text { practices, } 75 \\
\text { patients. }\end{array}$ & $\begin{array}{l}\text { Sig reduction in BZ use in both the letter group and the } \\
\text { consultation group compared to no change in control } \\
\text { group (data not reported). }\end{array}$ & $\begin{array}{l}6 \text { months to } \\
1 \text { year post } \\
\text { intervention }\end{array}$ \\
\hline $\begin{array}{l}\text { Cormack et al, } \\
1994 \text { [23] }\end{array}$ & England & $\begin{array}{l}\text { Long term BZ users (> } 6 \text { months) allocated to: } \\
1=\text { letter from GP to recommending stopping BZs } \\
2=\text { letter from GP + monthly advice sheets. } \\
3=\text { control }\end{array}$ & $\begin{array}{l}\mathrm{CT} \\
3 \text { practices } 209 \\
\text { patients. }\end{array}$ & $\begin{array}{l}30 \% \text { reduction in BZ use by intervention groups (sig } \\
\text { difference from control) No sig difference between } \\
\text { interventions. }\end{array}$ & $\begin{array}{l}6 \text { months post } \\
\text { intervention }\end{array}$ \\
\hline $\begin{array}{l}\text { Gorgels et al, } \\
2005 \text { [59] }\end{array}$ & Netherlands & $\begin{array}{l}1 \text { = discontinuation letter to long term users then } \\
\text { approximately } 3 \text { months after the letter, an invitation for } \\
\text { GP evaluation of BZ use. } 2=\text { control. }\end{array}$ & $\begin{array}{l}\text { RCT } \\
30 \text { practices } \\
1707 \text { patients } \\
\text { (intervention) and } \\
1821 \text { (control). }\end{array}$ & $\begin{array}{l}\text { Sig use reduction of BZ prescriptions in intervention group } \\
(24 \%) \text { vs. control (5\%). Sig reduction in BZ use in the } \\
\text { intervention group for those who attended the GP } \\
\text { evaluation (35\%) vs. those who did not go (24\%). }\end{array}$ & $\begin{array}{l}21 \text { months } \\
\text { post } \\
\text { intervention }\end{array}$ \\
\hline $\begin{array}{l}\text { Morrison, } \\
1990[60]\end{array}$ & UK & $\begin{array}{l}\text { Long term BZ users ( }>6 \text { months) were informed that they } \\
\text { should stop BZs by their GP. If agreed an individual plan } \\
\text { (how to decrease and number of follow-up visits) was } \\
\text { established. }\end{array}$ & $\begin{array}{l}\text { Convenience Sampling } \\
1 \text { practice, } 27 / 72 \\
\text { eligible patients } \\
\text { agreed. }\end{array}$ & $\begin{array}{l}\text { No new long term users started during study. } 37.5 \% \text { quit } \\
33.3 \% \text { reduced dose by }>50 \% \text { and } 15 \% \text { remained same } \\
\text { dose or }<50 \% \text { reduction. No-one increased their dose } \\
\text { during the study period. }\end{array}$ & $\begin{array}{l}1 \text { year (end of } \\
\text { study) }\end{array}$ \\
\hline $\begin{array}{l}\text { Onyett and } \\
\text { Turpin, } 1988 \text { [28] }\end{array}$ & UK & $\begin{array}{l}\text { Recruitment of long term users by notices. Asked to self- } \\
\text { reduce BZs then all participants received a pamphlet and } \\
\text { allocated to: } 1 \text { = group session. } 2 \text { = individual GP } \\
\text { appointment. }\end{array}$ & $\begin{array}{l}\text { Prospective cohort } \\
18 \text { patients. }\end{array}$ & $\begin{array}{l}59 \% \text { reduction in dose (group) and } 69 \% \text { reduction in dose } \\
\text { (GP meeting) but no sig difference between groups. }\end{array}$ & $\begin{array}{l}15 \text { week post } \\
\text { intervention }\end{array}$ \\
\hline $\begin{array}{l}\text { Brymer and } \\
\text { Rusnell, } \\
2000 \text { [27] }\end{array}$ & Canada & $\begin{array}{l}\text { Home assessment by nurse to determine if patients were } \\
\text { substance dependent. Saw Geriatrician for medical review, } \\
\text { who recommended a treatment plan (also sent to their } \\
\text { GP). Were encouraged to join support/educational groups. }\end{array}$ & $\begin{array}{l}\text { Observational study } \\
55 \text { elderly patients. }\end{array}$ & $\begin{array}{l}\text { Significant reduction of BZ use (59\%) between pre and post } \\
\text { intervention. }\end{array}$ & $\begin{array}{l}6 \text { months post } \\
\text { intervention }\end{array}$ \\
\hline Study & Location & $\begin{array}{l}\text { Intervention Design: } \\
\text { Educational Interventions Targeting GPs }\end{array}$ & $\begin{array}{l}\text { Study Design and } \\
\text { Size }\end{array}$ & Result & Follow up \\
\hline $\begin{array}{l}\text { Zwar et al, } \\
2000 \text { [61] }\end{array}$ & Australia & $\begin{array}{l}1 \text { = face to face } 20 \text { min educational visit by a GP focusing } \\
\text { on the management of long term users of BZs }+ \\
\text { guidelines + leaflets on relaxation techniques for patients. } \\
2=\text { control. }\end{array}$ & $\begin{array}{l}\text { RCT } \\
157 \text { GP registrars }\end{array}$ & $\begin{array}{l}\text { Sig decrease in overall BZs use in both groups ( } 0.6 \text { per } \\
100 \text { encounters for both group), however no difference } \\
\text { between control and intervention groups. }\end{array}$ & $\begin{array}{l}3 \text { practice } \\
\text { surveys - } \\
6 \text { monthly }\end{array}$ \\
\hline
\end{tabular}


Table 1 Educational Interventions to decrease benzodiazepine use (Continued)

\begin{tabular}{|c|c|c|c|c|c|}
\hline $\begin{array}{l}\text { Smith et al, } \\
1998 \\
{[30]}\end{array}$ & $\begin{array}{l}\text { Washington, } \\
\text { USA }\end{array}$ & $\begin{array}{l}1 \text { = mailed intervention package (guidelines, letter, } \\
\text { prescriber-specific profile, patient profile) for prescribers of } \\
\text { over users ( } 1 \text { tab per day }>1 \text { year) } \\
2=\text { control. }\end{array}$ & $\begin{array}{l}\text { RCT } \\
\text { GPs or pharmacist of } \\
222 \text { over users }\end{array}$ & $\begin{array}{l}\text { Sig decrease in BZ prescribing/dose for the intervention } \\
\text { group (27.6\%) compared to control (8.5\%). }\end{array}$ & $\begin{array}{l}3 \text { months post } \\
\text { intervention }\end{array}$ \\
\hline $\begin{array}{l}\text { Holm, } 1990 \\
{[32]}\end{array}$ & $\begin{array}{l}\text { Aarhus, } \\
\text { Denmark }\end{array}$ & $\begin{array}{l}1=\text { invite to a meeting on correct use of hypnotics/ } \\
\text { sedatives + educational material given at the meeting. } 2= \\
\text { mailed information on correct use and feedback on their } \\
\text { prescribing rate compared with others. } 3=\text { control. }\end{array}$ & $\begin{array}{l}\text { RCT } \\
356 \text { GPs } \\
\text { (245 practices) }\end{array}$ & $\begin{array}{l}\text { Sig decrease }(-53) \text { in DDD/1000 pat/week between pre and } \\
\text { post intervention. No difference between groups } 1 \text { and } 2 \\
\text { but sig difference between groups } 2 \text { and } 3 \text { ( } 2 \text { prescribed } \\
\text { more). }\end{array}$ & $\begin{array}{l}1-2 \text { months } \\
\text { post } \\
\text { intervention }\end{array}$ \\
\hline $\begin{array}{l}\text { De Burgh et al, } \\
1995 \text { [29] }\end{array}$ & $\begin{array}{l}\text { NSW, } \\
\text { Australia }\end{array}$ & $\begin{array}{l}1=20 \text { min educational visit to GPs. Educational material } \\
\text { left (management guidelines, review cards for long term } \\
\text { users). Offered access to sleep aids. Recommended to } \\
\text { review } 5 \text { patients and received a follow-up phone call. } 2 \text { = } \\
\text { control. }\end{array}$ & $\begin{array}{l}\text { RCT } \\
286 \text { GPS }\end{array}$ & $\begin{array}{l}\text { An overall decrease in BZ prescribing ( } 23.7 \%) \text {. No sig } \\
\text { difference between intervention and control for reduction } \\
\text { rate of BZ. Sig decrease between intervention ( } 72 \% \\
\text { decrease) and control ( } 13 \% \text { decrease) for rate of new BZ } \\
\text { scripts for insomnia. }\end{array}$ & $\begin{array}{l}\text { Approx } \\
6 \text { months post } \\
\text { intervention }\end{array}$ \\
\hline $\begin{array}{l}\text { Midlov et al, } \\
2006[62]\end{array}$ & $\begin{array}{l}\text { Skane, } \\
\text { Sweden }\end{array}$ & $\begin{array}{l}1=\text { two educational visits from pharmacist and GP } \\
\text { focusing on effects of medium and long acting BZs in the } \\
\text { elderly. } 2 \text { = control (received intervention after the study). }\end{array}$ & $\begin{array}{l}\text { RCT } \\
15 \text { GPs (8 in } \\
\text { intervention, } 7 \text { in } \\
\text { control) }\end{array}$ & $\begin{array}{l}\text { Sig decreases in all BZ prescribing (26.63\%) and } 25.8 \% \\
\text { decrease of long and medium acting BZs vs. control. }\end{array}$ & $\begin{array}{l}1 \text { year post } \\
\text { intervention }\end{array}$ \\
\hline $\begin{array}{l}\text { Berings et al, } \\
1994 \text { [31] }\end{array}$ & $\begin{array}{l}\text { East and } \\
\text { West } \\
\text { Flanders, } \\
\text { Belgium }\end{array}$ & $\begin{array}{l}1=\text { educational advertisement like mailings (with slogan) }+ \\
\text { educational visit. } 2=\text { mailings only } 3=\text { control }\end{array}$ & $\begin{array}{l}\text { RCT } \\
128 \text { physicians }\end{array}$ & $\begin{array}{l}\text { Sig decrease for whole sample pre to post intervention. Sig } \\
\text { decrease in BZ prescriptions between intervention } 1(24 \%) \\
\text { and control (3\%) and intervention } 2(14 \%) \text { and control and } \\
\text { intervention } 1 \text { and } 2 \text {. }\end{array}$ & $\begin{array}{l}4 \text { weeks post } \\
\text { intervention }\end{array}$ \\
\hline Study & Location & $\begin{array}{l}\text { Intervention Design: Educational Interventions Targeting } \\
\text { LTC }\end{array}$ & $\begin{array}{l}\text { Study Design and } \\
\text { Size }\end{array}$ & Result & Follow up \\
\hline $\begin{array}{l}\text { Hagen et al, } \\
2005[63]\end{array}$ & Alberta & $\begin{array}{l}1 \text { = Algorithm on non-pharmacological approaches for } \\
\text { agitation. Education based on algorithm to nurses, } \\
\text { pharmacists, or family members. } 30 \text { min education session } \\
\text { for GP. } 2 \text { = Control }\end{array}$ & $\begin{array}{l}C T \\
24 \text { LTC facilities, } \\
12 \text { (intervention) and } \\
12 \text { (control) }\end{array}$ & $\begin{array}{l}\text { BZ use in both the control and the intervention increased } \\
\text { post intervention (Sig increase for control only). At } 6 \\
\text { months post intervention total BZ use sig higher in control } \\
\text { vs. intervention. }\end{array}$ & $\begin{array}{l}\text { Every } \\
2 \text { months until } \\
6 \text { months post } \\
\text { intervention }\end{array}$ \\
\hline $\begin{array}{l}\text { Avorn et al, } \\
1992[64]\end{array}$ & Massachusetts & $\begin{array}{l}1=\text { physicians received } 3 \times \text { drug advertisement like } \\
\text { summaries of literature about geriatric medicine, } \\
\text { psychopharmacology }+3 \text { face to face visits to each doc by } \\
\text { pharmacist. } 4 \text { training sessions for nurses on alternatives to } \\
\text { psychoactive drugs }+ \text { ADRs. } 2 \text { = Control }\end{array}$ & $\begin{array}{l}\text { MPR } \\
12 \text { LTC facilities } 6 \\
\text { (intervention) and } 6 \\
\text { (control) both groups } \\
\text { were matched }\end{array}$ & $\begin{array}{l}\text { Sig difference in the \% change to more appropriate BZs in } \\
\text { intervention (64\%) vs. control (4\%). E.g. long acting to short } \\
\text { acting. }\end{array}$ & $\begin{array}{l}30 \text { days post } \\
\text { intervention }\end{array}$ \\
\hline $\begin{array}{l}\text { Schmidt et al, } \\
1998 \text { [65] }\end{array}$ & Sweden & $\begin{array}{l}1=\text { Monthly meeting for } 12 \text { months led by pharmacist and } \\
\text { included physician, and nurses. Each patient's medications } \\
\text { were reviewed. } 2 \text { = Control }\end{array}$ & $\begin{array}{l}\text { RCT } \\
33 \text { nursing homes ( } 15 \\
\text { intervention, } \\
18 \text { control) }\end{array}$ & $\begin{array}{l}\text { Sig increase (from baseline) in prescribing of appropriate } \\
\text { hypnotics }(+70 \%) \text { and anxiolytics }(+50 \%) \text { in intervention } \\
\text { group. No sig difference in control group }\end{array}$ & $\begin{array}{l}1 \text { month post } \\
\text { intervention }\end{array}$ \\
\hline $\begin{array}{l}\text { Gilbert et al, } \\
1993[36]\end{array}$ & $\begin{array}{l}\text { Adelaide, } \\
\text { Australia }\end{array}$ & $\begin{array}{l}1=\text { letter to residents inviting participation in relaxation } \\
\text { groups }(8 \times 40 \mathrm{~min}) \text { audio tape of relaxation for practice } \\
\text { and information about sleep \& anxiety medications. Nurses } \\
\text { received a seminar on dealing with BZ withdrawal. Doctors } \\
\text { received letter of progress. } 2 \text { = Control }\end{array}$ & $\begin{array}{l}\text { Prospective cohort } \\
2 \text { LTC facilities }\end{array}$ & $\begin{array}{l}\text { There was a sig decrease in the } \% \text { of BZs users' from } \\
\text { baseline (70\%) to post intervention (35\%). No change in } \\
\text { control }\end{array}$ & $\begin{array}{l}12 \text { weeks after } \\
\text { baseline }\end{array}$ \\
\hline
\end{tabular}


Table 2 Audit and Feedback Interventions to decrease benzodiazepine use

\begin{tabular}{|c|c|c|c|c|c|}
\hline Study & Location & $\begin{array}{l}\text { Intervention Design: Audit and Feedback } \\
\text { Interventions targeting GPs }\end{array}$ & Study Design and Size & Result & Follow up \\
\hline Baker et al, 1997 [66] & Leicester, UK & $\begin{array}{l}\text { Audit on all long-term users ( }>4 \text { weeks) in } \\
\text { the medical centre then GPs received either: } \\
1=\text { feedback on prescribing practices }+ \\
\text { criteria for the management of long term } \\
\text { BZ users. } 2 \text { = feedback + criteria + reminder } \\
\text { cards for patient files. }\end{array}$ & $\begin{array}{l}\text { RT } \\
18 \text { practices patients }=2409 \\
\text { long term BZ users }\end{array}$ & $\begin{array}{l}\text { Both groups changed after intervention with } \\
\text { respect to levels of compliance to criteria. } \\
8.2 \% \text { of patients were stopped and } 1.3 \% \\
\text { were decreasing BZs. No difference between } \\
\text { groups. }\end{array}$ & $\begin{array}{l}2^{\text {nd }} \text { audit completed } 1 \text { year } \\
\text { post intervention }\end{array}$ \\
\hline $\begin{array}{l}\text { Holden et al, } 1994 \\
\text { [67] }\end{array}$ & $\begin{array}{l}\text { Liverpool, } \\
\text { Southport - UK }\end{array}$ & $\begin{array}{l}\text { Audit of BZ use + GPs invited to } 2 \text { meetings } \\
\text { on auditing BZ use in general practice. } \\
\text { Individual practices determined their own } \\
\text { BZ policy for prescribing and reducing use. }\end{array}$ & $\begin{array}{l}\text { Observational } \\
15 \text { practices, } 3234 \text { patients }\end{array}$ & $\begin{array}{l}\text { Overall reduction of } 16 \% \text {. Sig reduction in } \\
\text { those }<65(25 \%) \text { compared to those } \\
>65=12 \% .\end{array}$ & $\begin{array}{l}2^{\text {nd }} \text { audit at } 8 \text { months (end } \\
\text { of study) }\end{array}$ \\
\hline $\begin{array}{l}\text { Pimlott et al, } 2003 \\
\text { [68] }\end{array}$ & Canada - Ontario & $\begin{array}{l}1=\text { audit and feedback on GPs prescribing } \\
\text { of BZs compared to peers and best } \\
\text { practice + information } \\
\text { sheet on BZs every } 2 \text { months for } 6 \text { months. } \\
2=\text { Control group had the same } \\
\text { intervention for antihypertensives. }\end{array}$ & $\begin{array}{l}\text { RCT } \\
168 \text { GPs (intervention) } 206 \\
\text { GPs (control) }\end{array}$ & $\begin{array}{l}\text { No sig decrease in BZ prescribing and no } \\
\text { sig difference between intervention and } \\
\text { control groups. }\end{array}$ & 6 months post intervention \\
\hline Study & Location & $\begin{array}{l}\text { Intervention Design: Audit and Feedback } \\
\text { Targeting LTC }\end{array}$ & Study Design and Size & Result & Follow up \\
\hline $\begin{array}{l}\text { McClaugherty, } 1997 \\
\text { [69] }\end{array}$ & Texas & $\begin{array}{l}\text { LTC pharmacist audited BZ use + gave } \\
\text { feedback to } \\
\text { nurses and doctors. Nurses were given sleep } \\
\text { promoting guidelines. OT's \& physio's were } \\
\text { encouraged to increase activities for those } \\
\text { who couldn't sleep. }\end{array}$ & $\begin{array}{l}\text { Quasi-Experimental } \\
10 \text { Nursing Homes, } 3 \text { Texas } \\
\text { counties }\end{array}$ & $\begin{array}{l}\text { \% of patients prescribed routine BZ } \\
\text { decreased from } 4.5 \% \text { (baseline) to } 1.6 \% \\
\text { (post intervention). \% of patients prescribed } \\
\text { BZ on an as needed basis increased from } \\
7.9 \% \text { (baseline) to } 9.3 \% \text { (post intervention) }\end{array}$ & 3 months post intervention \\
\hline $\begin{array}{l}\text { Gill et al, } \\
2001[70]\end{array}$ & Ontario, Canada & $\begin{array}{l}\text { Review of patients chart }+ \text { a letter was sent } \\
\text { to the treating doctor if inappropriate e.g. } \\
\text { long acting BZ explaining why medication } \\
\text { was inappropriate and suggestions for } \\
\text { alternative therapy. }\end{array}$ & $\begin{array}{l}\text { Quasi-Experimental } \\
1 \text { LTC facility, } 450 \text { Patients }\end{array}$ & $\begin{array}{l}37.9 \% \text { of inappropriate medications were } \\
\text { withdrawn or changed after the letter. }\end{array}$ & $\begin{array}{l}2 \text { months after follow-up } \\
\text { letters }\end{array}$ \\
\hline Elliot et al, 2001 [37] & Australia & $\begin{array}{l}\text { Audit and } 1 \mathrm{~h} \text { meeting }=\text { feedback to all } \\
\text { staff on prescribing compared to other } \\
\text { hospitals and review of literature }+ \text { posters } \\
\text { in wards }\end{array}$ & $\begin{array}{l}\text { Quasi-Experimental } \\
9 \text { hospitals ( } 6 \text { aged care } 3 \\
\text { medical wards) }\end{array}$ & $\begin{array}{l}\text { No sig reduction in BZ use. Sig increase in } \\
\text { appropriate prescribing at } 8 \text { week (22\%) and } \\
6 \text { months (30\%) post intervention. }\end{array}$ & $\begin{array}{l}\text { 4-8 weeks (all) and } 6 \text { months } \\
\text { (for } 3 \text { hospitals only) post } \\
\text { intervention }\end{array}$ \\
\hline Study & Location & $\begin{array}{l}\text { Intervention Design: } \\
\text { Audit and Feedback + Education } \\
\text { Targeting LTC }\end{array}$ & Study Design and Size & Result & Follow up \\
\hline $\begin{array}{l}\text { Roberts et al, } 2001 \\
\text { [34] }\end{array}$ & $\begin{array}{l}\text { QLD + NSW, } \\
\text { Australia }\end{array}$ & $\begin{array}{l}1=11 \text { hrs of problem based education } \\
\text { session for nurses + wall charts, bulletins, } \\
\text { telephone, visits. Written drug review for } \\
500 \text { selected patients. Report on review } \\
\text { placed in patient's records and available to } \\
\text { the GPs. } \\
2=\text { Control }\end{array}$ & $\begin{array}{l}\text { RCT } \\
52 \text { nursing homes, } 13 \\
\text { (intervention), } 39 \text { (control). }\end{array}$ & $\begin{array}{l}\text { Sig difference in the reduction of BZs } \\
\text { between intervention (decreased } 597 \text { items/ } \\
\text { year/ } 1000 \text { residents) and control (increased } \\
278 \text { items/year/1000 residents). }\end{array}$ & 12 months (end of study) \\
\hline
\end{tabular}


Table 2 Audit and Feedback Interventions to decrease benzodiazepine use (Continued)

\begin{tabular}{|c|c|c|c|c|c|}
\hline Batty et al, 2001 [35] & $\begin{array}{l}\text { England/ } \\
\text { Wales }\end{array}$ & $\begin{array}{l}\text { Audit then: } \\
1=\text { lecture to staff on literature review on } \\
\text { appropriate prescribing of BZs. Feedback on } \\
\text { prescribing compared to another hospitals. } \\
2=\text { Bulletin ( } 2 \text { sided A4) with same } \\
\text { information as lecture. } \\
3=\text { Control }\end{array}$ & $\begin{array}{l}\text { RCT } \\
\text { Elderly inpatients at } \\
17 \text { hospitals } \\
\text { ( } 6 \text { lecture, } 4 \text { bulletin, } \\
7 \text { control) }\end{array}$ & $\begin{array}{l}\text { No sig change in any group but verbal } \\
\text { group increased appropriate prescribing by } \\
15 \% \text {, bulletin decreased appropriate } \\
\text { prescribing (9\%) and control remained the } \\
\text { same. }\end{array}$ & 4-6 weeks post intervention \\
\hline $\begin{array}{l}\text { Eide and Schjott, } \\
2001 \text { [33] }\end{array}$ & Norway & $\begin{array}{l}1 \text { = Audit of BZ use, feedback to staff } \\
\text { (reports and a presentation). Academic } \\
\text { education to all staff by pharmacist, } \\
\text { consisting of } 6 \text { simple rules for the use of } \\
\text { hypnotics (data collected in } 1995 \text { and 2000). } \\
2 \text { = Control (data collected in } 2000 \text { only) }\end{array}$ & $\begin{array}{l}\text { CT } \\
10 \text { LTC Facilities, } \\
5 \text { (intervention) and } \\
5 \text { (control) }\end{array}$ & $\begin{array}{l}\text { Sig dif in the \% of patients use BZS in } \\
\text { control (44\%) compared to intervention } \\
(24 \%) \text { post intervention. Sig higher dose of } \\
\text { BZs in intervention group in } 2000(60 \%) \\
\text { compared to } 1995 \text { (38\%). }\end{array}$ & 5 years post intervention \\
\hline $\begin{array}{l}\text { Crotty et al, } 2004 \\
\text { [71] }\end{array}$ & $\begin{array}{l}\text { Adelaide, } \\
\text { Australia }\end{array}$ & $\begin{array}{l}\text { Audit then: } \\
1=\text { GP received education and guidelines } \\
\text { and audit of use. Nurses received education } \\
\text { in behaviour management and all staff } \\
\text { received education on reducing } \\
\text { psychotropic medication use } \\
2=\text { control }\end{array}$ & $\begin{array}{l}\text { MRP } \\
20 \text { LTC facilites, } \\
10 \text { (intervention) and } \\
10 \text { (control) }\end{array}$ & $\begin{array}{l}\text { No sig reduction in BZ use }(6.3 \% \text {, } \\
\text { intervention, } 0 \% \text { control), no significant } \\
\text { decrease in long acting BZs ( } 2.8 \% \\
\text { intervention and } 0.9 \% \text { control) and no sig } \\
\text { difference in BZ being prescribed on a as } \\
\text { needed basis ( } 4 \% \text { intervention and } 1 \% \\
\text { control) }\end{array}$ & $\begin{array}{l}2^{\text {nd }} \text { audit was at } 7 \text { months } \\
\text { (end of study) }\end{array}$ \\
\hline
\end{tabular}

BZ = benzodiazepine, GP = General Practitioner, Av = Average, Sig = statistically significant $(p<0.05), \mathrm{LTC}=$ long term care, OT's $=$ Occupational Therapists, RCT $=$ Randomized controlled trial, $\mathrm{CT}=\mathrm{Controlled}$ trial, $\mathrm{RT}=$ randomized trial, $\mathrm{MPR}=$ Matched pair randomisation 
Table 3 Alert Interventions to decrease benzodiazepine use

\begin{tabular}{|c|c|c|c|c|c|}
\hline Study & Location & $\begin{array}{l}\text { Intervention Design: } \\
\text { Alert Interventions targeted at GPs }\end{array}$ & $\begin{array}{l}\text { Study Design } \\
\text { and Size }\end{array}$ & Result & Follow up \\
\hline $\begin{array}{l}\text { Simon et al, } \\
2006[72]\end{array}$ & $\begin{array}{l}\text { USA - } \\
\text { Oregon \& } \\
\text { Washington }\end{array}$ & $\begin{array}{l}1=\text { age Specific (> } 65 \text { years) alert for } \\
\text { long acting BZs. } 2=\text { alerts }+ \text { academic } \\
\text { detailing (group education }+ \\
\text { follow-up letter). }\end{array}$ & $\begin{array}{l}\text { Cluster } \\
\text { randomized trial } \\
239 \text { clinicians }\end{array}$ & $\begin{array}{l}\text { No sig decrease in prescribing to } \\
\text { elderly. No difference between alerts } \\
\text { (decrease of } 3.0 \text { dispensed medications } \\
\text { per } 10,000 \text { members) and alerts }+ \\
\text { academic detailing (decrease of } 19.7 \\
\text { dispensed medications per } 10,000 \text { ) }\end{array}$ & $\begin{array}{l}18 \text { months } \\
\text { post } \\
\text { intervention }\end{array}$ \\
\hline $\begin{array}{l}\text { Monane et al, } \\
1998 \text { [73] }\end{array}$ & America & $\begin{array}{l}1=\text { age specific (> } 65 \text { years) alert } \\
\text { system at pharmacy (mail order and } \\
\text { retail). If medication is deemed to be } \\
\text { inappropriate then conversation } \\
\text { between pharmacist and prescriber } \\
\text { occurred. } 2 \text { = control. }\end{array}$ & $\begin{array}{l}\text { Population based } \\
\text { cohort } \\
2.3 \text { million } \\
\text { people }>65 \text { years } \\
\text { filled a script } \\
\text { during study } \\
\text { period }\end{array}$ & $\begin{array}{l}\text { Sig difference between intervention } \\
\text { ( } 40 \% \text { of cases) and control (2\%) for the } \\
\text { change of prescriptions for long acting } \\
\text { BZs. Sig difference between control (2\%) } \\
\text { and intervention ( } 25 \% \text { ) for change to } \\
\text { prescriptions of short acting BZs that } \\
\text { exceeded the maximum daily dose. }\end{array}$ & $\begin{array}{l}1 \text { year study } \\
\text { (measured } \\
\text { total number } \\
\text { of changes } \\
\text { over } \\
1 \text { year) }\end{array}$ \\
\hline
\end{tabular}

$\mathrm{BZ}=$ benzodiazepine, $\mathrm{Sig}=$ statistically significant $(\mathrm{p}<0.05), \mathrm{GP}=$ General Practitioner

It is from these critiques that factors influencing success or otherwise can be determined, and lessons about future study design learnt.

\section{Risk of Bias}

Some intervention studies about benzodiazepines may have been excluded from this commentary due to the use of the selected key words, however it was difficult to be more general than the terms selected without generating a totally non-specific (and much larger) database of possible articles. There may be a publication bias, as studies are known to be less reported if they generate negative results (or 'no change') with non-completion of the intervention or write up more likely.

\section{Results}

The search of the databases returned 8437 studies (Figure 1). Of these, 32 studies fulfilled all the predetermined inclusion criteria and could be categorized as described above. For each study the data were extracted for the following characteristics; location of the intervention, intervention design, study type, participant numbers, intervention follow-up time and study outcomes. Comparisons of these characteristics are described below.

\section{Educational Interventions}

The 19 studies (summarized in Table 1) have been targeted at 3 different groups; those aimed at GPs, at consumers and at long term care facilities.

\section{Audit and Feedback Interventions}

The 10 studies (summarized in Table 2) were classified into 2 categories, those aimed at GPs and those aimed at long term care facilities. A sub category was also identified within the interventions aimed at long term care facilities, those which include education in addition to audit and feedback.

\section{Alert Interventions}

These 2 studies (summarized in Table 3) were only targeted at GPs.

The final study included was a multifaceted intervention which used an educational approach to target GPs, pharmacists and consumers in a rural region of South Australia. A 19\% reduction in benzodiazepine use was maintained 2 years after the intervention finished [24].

The critiques of the 32 studies (Table 1, 2 and 3) are discussed below.

\section{Education}

\section{Targeted at Consumers}

Consumer focused educational intervention specifically targeted individual long term benzodiazepine users rather than an overall public health approach or community awareness campaigns and used mail-outs as the main approach. In all of these studies, long term benzodiazepine users were sent letters from a GP, or in one case from a pharmacist [25]. The letters asked that patients cut back on the benzodiazepines and gave basic advice on how to do this (e.g. gradually reducing use over a period of weeks). All of these studies saw a similar dose reduction (22\% - 30\%). Studies which invited participants to visit a GP, during which consultation they were asked to reduce their benzodiazepine dose face to face, resulted in significant decreases in benzodiazepine doses compared to the control but did not significantly differ to those who received only the letter $[23,26]$. This demonstrates that a simple bulk mail-out to identified individuals approach appears to be just as effective in improving use of benzodiazepines, and may have advantages compared to the more time consuming, 


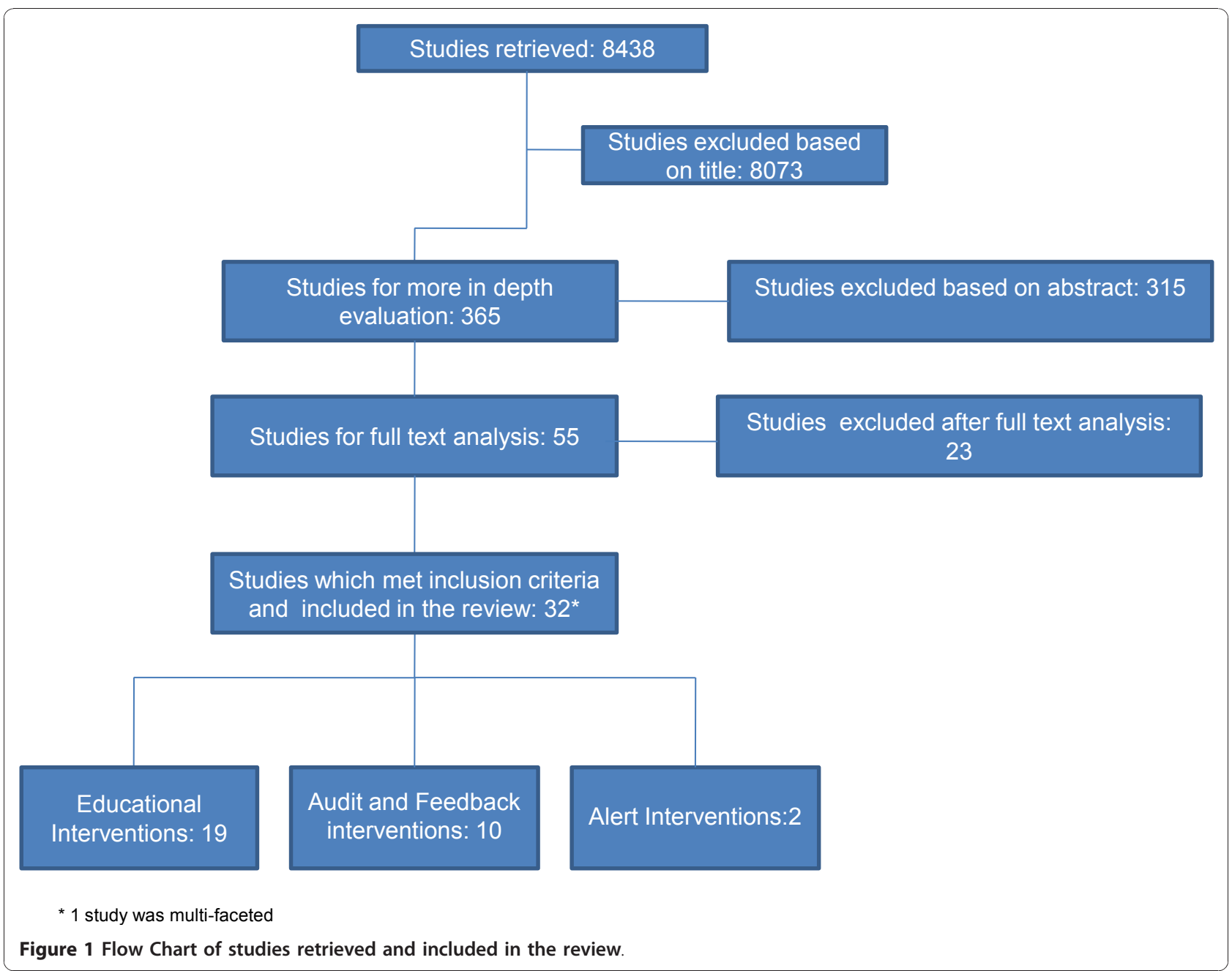

and expensive approach of educational sessions or GP consultations.

Educational and support group sessions were also used as an educational method. The studies using group sessions did see larger reductions (69\% and 59\% dose reduction $[27,28]$ respectively) in benzodiazepine use compared to the mail-out studies but neither of these studies used a control group so conclusions are difficult to draw.

\section{Targeted at GPs}

Education for GPs included packages containing benzodiazepine prescribing guidelines, face to face visits and 'promotional' material with key messages.

When compared to control, $50 \%$ of the educational interventions targeted at GPs had significant decreased benzodiazepine use and 17\% (1 study, [29]) which did not reduce overall use of benzodiazepines did significantly decrease the number of new patients being prescribed benzodiazepines.
The study [30] that saw the largest reduction in benzodiazepine use differed from the other GP targeted educational studies as it did not rely upon voluntary participation. Any GP who prescribed to a Washington State Medicaid beneficiary deemed to be a "high utilizer" of benzodiazepines was sent the intervention package (see Table 1 for further details). In the other studies the voluntary recruitment design may have resulted in the participation of GPs who prescribed benzodiazepines more rationally (pre-intervention) than non-participating GPs. If this occurred, smaller changes between intervention (pre and post) and control groups would be seen, and this selection bias could be influencing the conclusions.

Differences in the delivery of the education (mail vs. personal visit) had varying effects on the "success" of the studies. For example, Smith et al, 1998 (the study with the largest decrease in benzodiazepine use) delivered education via mail-out. This success is supported by a study which had a mail-out and educational visit 
intervention arms [31]. They found a significant difference in the decrease of benzodiazepine use between control and both the educational visit (24\%) and the mail out (14\%) arms and between the two interventions. However Holm,1990 found no significant difference between the education visit and the mail-out arms of their intervention [32].

\section{Targeted at long term care facilities}

The educational tools used in long term care facilities varied from prescribing algorithms and bulletins to staff meetings and lectures.

Educational interventions delivered to long term care facilities differed compared to those delivered to GPs and consumers in two ways. Firstly, some studies included audit and feedback in addition to education [33-35] and all of the studies were multi faceted (the education was directed at a variety of people, including nurses, GPs, family members, pharmacists) rather than just targeting GPs. This multifaceted approach could in some part be responsible for the larger percentage of studies which either decreased use or reported improved prescribing of benzodiazepine in this setting when compared to the GP targeted studies.

The majority of the long term care facility educational studies used pharmacists to deliver the "educational message" to staff, (one study did not specify [34] and 1 used a psychologist [36]). Of the 5 pharmacist led studies two were unsuccessful in either decreasing benzodiazepine use or increasing appropriate prescribing suggesting that, in this context, the type of health professional delivering the education does not determine the success of the intervention.

\section{Audit and feedback}

The audit and feedback studies which targeted long term care facilities involved more than 1 health professional e.g. doctors, nurses, physiotherapists and pharmacists whereas the GP targeted interventions only involved the GPs. This may be responsible for the difference in results between the 2 groups, as the audit and feedback studies in long term care facilities all improved appropriateness of prescribing (as defined by each study) but only 1 of the 3 GP studies achieved this same success. Audit and feedback may be resisted by some prescribers, which may explain perhaps why better results for benzodiazepine prescribing were obtained in long term care facility audits, where multiple different health practitioners were targeted compared to the audits of individual GPs alone.

Another factor that appears to affect the outcome of the interventions is how the feedback was delivered. All studies which successfully achieved their aim (reducing or improving use of benzodiazepines) gave feedback orally, either in a meeting or individual consultations instead of just mailing.

Both success and failure to improve benzodiazepine prescribing was seen in both the audit and feedback intervention group [37-39] and the audit and feedback in conjunction with education group [33-35,40]. It can therefore not be determined if education in addition to audit and feedback interventions improves the outcome of the study.

\section{Alerts}

The GP targeted decision support tool alerted GPs when they were either prescribing a long acting benzodiazepine to a patient over 65 or initiating a patient on a benzodiazepine. This intervention found no difference between intervention and control when measuring the change in the target medications per 10,000 patients per quarter. This is not surprising as GPs are renowned for turning the electronic reminders/alerts off or ignoring all alerts that appear [41].

The second alert system was run through the pharmacy, where the pharmacist contacted the GP to discuss the prescription if an alert appeared. This study did see a significant increase in the appropriate prescribing of benzodiazepines (as determined by an independent advisory board and the use of Beers Criteria) [20]. An improvement was defined as a decrease in number of benzodiazepine prescriptions which, a) exceeded therapeutic dose, b) prescribed to the over $65 \mathrm{~s}$ and c) contained long acting benzodiazepines.

With both studies having a similar definition of "appropriate", the difference here appears to be the pharmacist involvement. It has been suggested that community pharmacists are an underused resources in prescribing studies [42], therefore it may be an appropriate time to incorporate pharmacists into future studies, as they are fast becoming one of the most utilized health professionals in many countries [43].

\section{Discussion}

\section{Summary of Evidence}

This investigation solely focuses on interventions designed to improve prescribing and/or use of benzodiazepines and related drugs in the last 20 years and has demonstrated that many different interventions strategies are used worldwide, with varying success. The most successful interventions to improve prescribing and use of benzodiazepines were those which are multi-faceted, targeting a number of groups (prescribers as well as consumers).

\section{Limitations}

This study may be limited by the fact that this was not a complete systematic review and there are insufficient 
data available to conduct a meta-analysis on the effect size of any specific intervention, hence bias in interpretation may have been introduced by the non-quantitative summary techniques.

\section{Knowledge Translation}

The points below outline some aspects of the study designs that may enhance the success of any future intervention.

Points which appear to enhance success of an intervention

- Advise individual patients to stop/cut back on their benzodiazepines (either via letter or face-to-face). A follow-up consultation may improve the success for individual patients.

- Encourage consumer support groups, to enhance self-withdrawal of benzodiazepines by patients

- Encourage inter-professional approach e.g. pharmacists alerting prescribers of potentially inappropriate prescribing of benzodiazepines or nurses regularly contacting/providing information to patients who are trying to cut back on their benzodiazepines

- Use oral feedback rather than written in audit and feedback studies to GPs; consider group audit, such as through long term care facilities, rather than audit of individuals

- Educate all long term care staff about alternative methods to treat insomnia and cutting back on the use of benzodiazepines

\section{Conclusions}

One of the key success factors noted with all intervention approaches was the use of a multi-target design where a number of audiences or approaches were used; this may be why the consumer interventions worked well, as essentially they had two target audiences, the consumer and the GP or health professional involved in the study. This also agrees with findings from other therapeutic areas, for example a mass media campaign around appropriate use of antimicrobials and student taught programs for reducing antibiotic use during colds or flu $[9,44,45]$. The one distinctly multi-faceted study in this review of benzodiazepine interventions also saw a sustained reduction (19\%) in benzodiazepine use when all health professionals and the community were educated [24]. Conducting multi-faceted interventions can often be labor intensive, expensive and difficult to administer on a large scale basis and therefore can usually only be maintained for a limited time. Future interventions which use delivery methods such as email or websites may help to ameliorate some of these financial or 'reach' problems. This commentary has suggested that the use of support/ educational groups for consumers may improve the 'success' of an intervention, this could easily be administered using social networking technologies.

Whilst it is known that many healthcare providers have resisted using information technology due to privacy and infrastructure issues [46,47], many pharmacies, and medical centres now use the internet and email regularly and frequently [48-50]. Future investigation and validation into the effectiveness of electronic delivery method to a number of target groups could be beneficial, as varying results have been seen [51-54]. Studies which incorporate newer information technology may lead to more sustained changes as they are simple to administer, and can have a large scale impact by inexpensively involving a variety of health professionals and/ or consumers $[55,56]$.

\section{Acknowledgements}

A. Smith was supported by an Australian Postgraduate Award.

\section{Authors' contributions}

AS led the design and conduct of the study; interpretation of the results, and manuscript development. ST contributed to the interpretation of the results and manuscript development. All authors approved the final manuscript.

\section{Competing interests}

The authors declare that they have no competing interests.

Received: 2 July 2010 Accepted: 30 November 2010 Published: 30 November 2010

\section{References}

1. Aragones E, Pinol JL, Labad A, Folch S, Melich N: Detection and management of depressive disorders in primary care in Spain. Int J Psychiatry Med 2004, 34(4):331-343.

2. Goldney $\mathrm{RD}$, Fisher $\mathrm{L}$ : Use of prescribed medications in a South Australian community sample. Med J Aust 2005, 183(5):251-253.

3. Ohayon MM, Lader MH: Use of psychotropic medication in the general population of France, Germany, Italy, and the United Kingdom. J Clin Psychiatry 2002, 63(9):817-825.

4. Mant A, McManus P: Benzodiazepine use-continuing to fall. Med J Aust 1994, 161(7):454.

5. Mant A, Walsh RA: Reducing benzodiazepine use. Drug Alcohol Rev 1997, 16(1):77-84

6. Mant A, Whicker SD, McManus P, Birkett DJ, Edmonds D, Dumbrell D: Benzodiazepine utilisation in Australia: report from a new pharmacoepidemiological database. Aust J Public Health 1993, 17(4):345-349.

7. Smith A, Sketris I, Cooke C, Gardner D, Kisely S, Tett S: A comparison of benzodiazepines and related drug use in Nova Scotia, Canada and Australia. Can J Psychiat 2008, 53(8):545-552.

8. Dollman WB, Leblanc VT, Stevens L, O'Connor PJ, Roughead EE, Gilbert AL: Achieving a sustained reduction in benzodiazepine use through implementation of an area-wide multi-strategic approach. J Clin Pharm Ther 2005, 30(5):425-432.

9. Oxman AD, Thomson MA, Davis DA, Haynes RB: No magic bullets: a systematic review of 102 trials of interventions to improve professional practice. CMAJ 1995, 153(10):1423-1431.

10. Australian Medicines Handbook. [http://www.amh.hcn.net.au].

11. Antibiotic Writing Group: Therapeutic Guidelines: Antibiotic. Melbourne: Therapeutic Guidelines Limited; 200312 [http://www.tg.com.au/ip/ complete/]. 
12. Buffett-Jerrott SE, Stewart SH: Cognitive and sedative effects of benzodiazepine use. Curr Pharm Design 2002, 8(1):45-58.

13. Kaplan ME, DuPont RL: Benzodiazepines and anxiety disorders: a review for the practicing physician. Curr Med Res Opin 2005, 21(6):941-950.

14. French DD, Campbell R, Spehar A, Angaran DM: Benzodiazepines and injury: a risk adjusted model. Pharmacoepidem Dr S 2005, 14(1):17-24.

15. Paris J: Clinical practice guidelines. Management of anxiety disorders. Can J Psychiatry 2006, 51 (8 Suppl 2):9S-91S.

16. Cumming R, Le Couteur D: Benzodiazepines and risk of hip fractures in older people: a review of the evidence. CNS Drugs 2003, 17(11):825-837

17. Barker M, Greenwood K, Jackson M, Crowe S: Cognitive effects of longterm benzodiazepine use: a meta-analysis. CNS Drugs 2004, 18(1):37-48.

18. Grimshaw JM, Shirran L, Thomas R, Mowatt G, Fraser C, Bero L, Grilli R, Harvey E, Oxman A, O'Brien MA: Changing Provider Behavior: An Overview of Systematic Reviews of Interventions. Med Care 2001, 39(8): $\|2-\| 45$.

19. Ostini R, Hegney D, Jackson C, Williamson M, Mackson JM, Gurman K, Hall W, Tett SE: Systematic Review of Interventions to Improve Prescribing. Ann Pharmacother 2009, 43(3):502-513.

20. Fick DM, Cooper JW, Wade WE, Waller JL, Maclean JR, Beers MH: Updating the Beers criteria for potentially inappropriate medication use in older adults: results of a US consensus panel of experts. Arch Intern Med 2003, 163(22):2716-2724.

21. Gallagher PF, Barry PJ, Ryan C, Hartigan I, O'Mahony D: Inappropriate prescribing in an acutely ill population of elderly patients as determined by Beers' Criteria. Age Ageing 2008, 37(1):96-101.

22. Cabana MD, Rand CS, Powe NR, Wu AW, Wilson MH, Abboud PA, Rubin HR: Why don't physicians follow clinical practice guidelines? A framework for improvement.[see comment]. Jama 1999, 282(15):1458-1465.

23. Cormack MA, Sweeney KG, Hughes-Jones H, Foot GA: Evaluation of an easy, cost-effective strategy for cutting benzodiazepine use in general practice. Br J Gen Pract 1994, 44(378):5-8

24. Dollman WB, LeBlanc VT, Stevens L, O'Connor PJ, Roughead EE, Gilbert AL: Achieving a sustained reduction in benzodiazepine use through implementation of an area-wide multi-strategic approach. J Clin Pharm Ther 2005, 30(5):425-432.

25. Towle I, Adams J: A novel, pharmacist-led strategy to reduce the prescribing of benzodiazepines in Paisley. The Pharmaceutical Journal 2006, 276(Feb 4):136-138.

26. Heather N, Bowie A, Ashton H, McAvoy B, Spencer I, Brodie J, Giddings D: Randomised controlled trial of two brief interventions against long-term benzodiazepine use: outcome of intervention. Addict ResTheory 2004, 12(2):141-154

27. Brymer C, Rusnell I: Reducing substance dependence in elderly people: The side effects program. J Popul Ther Clin Pharmacol 2000, 7(3):161-166.

28. Onyett SR, Turpin G: Benzodiazepine withdrawal in primary care: A comparison of behavioural group training and individual sessions. Behav Psychother 1988, 16(4):297-312.

29. de Burgh S, Mant A, Mattick RP, Donnelly N, Hall W, Bridges-Webb C: A controlled trial of educational visiting to improve benzodiazepine prescribing in general practice. Aust J Public Health 1995, 19(2):142-148.

30. Smith DH, Christensen DB, Stergachis A, Holmes G: A randomized controlled trial of a drug use review intervention for sedative hypnotic medications. Med Care 1998, 36(7):1013-1021.

31. Berings $D$, Blondeel $L$, Habraken $H$ : The effect of industry-independent drug information on the prescribing of benzodiazepines in general practice. Eur J Clin Pharmacol 1994, 46(6):501-505.

32. Holm M: Intervention against long-term use of hypnotics/sedatives in general practice. Scand J Prim Health Care 1990, 8(2):113-117

33. Eide $E$, Schjott J: Assessing the effects of an intervention by a pharmacist on prescribing and administration of hypnotics in nursing homes. Pharm World Sci 2001, 23(6):227-231.

34. Roberts MS, Stokes JA, King MA, Lynne TA, Purdie DM, Glasziou PP, Wilson DA, McCarthy ST, Brooks GE, de Looze FJ, et al: Outcomes of a randomized controlled trial of a clinical pharmacy intervention in 52 nursing homes. Br J Clin Pharmacol 2001, 51(3):257-265.

35. Batty GM, Oborne CA, Hooper R, Jackson SHD: Investigating intervention strategies to increase the appropriate use of benzodiazepines in elderly medical in-patients. Br J Clin Govern 2001, 6(4):252-258.
36. Gilbert A, Owen N, Innes JM, Sansom L: Trial of an intervention to reduce chronic benzodiazepine use among residents of aged-care accommodation. Aust N Z J Med 1993, 23(4):343-347.

37. Elliott RA, Woodward MC, Oborne CA: Improving benzodiazepine prescribing for elderly hospital inpatients using audit and multidisciplinary feedback. Intern Med J 2001, 31(9):529-535.

38. Holden JD, Hughes IM, Tree A: Benzodiazepine prescribing and withdrawal for 3234 patients in 15 general practices. Fam Pract 1994, 11(4):358-362.

39. Pimlott NJG, Hux JE, Wilson LM, Kahan M, Li C, Rosser WW: Educating physicians to reduce benzodiazepine use by elderly patients: $\mathrm{A}$ randomized controlled trial. CMAJ 2003, 168(7):835-839.

40. Crotty M, Whitehead C, Rowett D, Halbert J, Weller D, Finucane P, Esterman A: An outreach intervention to implement evidence based practice in residential care: a randomized controlled trial [ISRCTN67855475]. BMC Health Serv Res 2004, 4(1):6.

41. Strom B: Pharmacoepidemiology. Chichester: John Wiley and Sons Ltd; 4 2005.

42. Majumdar SR, Soumerai SB: Why most interventions to improve physician prescribing do not seem to work. CMAJ 2003, 169(1):30-31.

43. Pharmacies Connecting the Community for Better Health. [http://www. guild.org.au/].

44. Lambert MF, Masters GA, Brent SL: Can mass media campaigns change antimicrobial prescribing? A regional evaluation study. J Antimicrob Chemother 2007, 59(3):537-543.

45. Cebotarenco N, Bush PJ: Reducing antibiotics for colds and flu: a studenttaught program. Health Educ Res 2008, 23(1):146-157.

46. Schattner P, Pleteshner $C$, Bhend $H$, Brouns J: Guidelines for computer security in general practice. Inform Prim Care 2007, 15(2):73-82.

47. Western MC, Dwan KM, Western JS, Makkai T, Del Mar C: Computerisation in Australian general practice. Aust Fam Physician 2003, 32(3):180-185.

48. Wall TC, Mian MA, Ray MN, Casebeer L, Collins BC, Kiefe Cl, Weissman N, Allison JJ: Improving physician performance through Internet-based interventions: who will participate? J Med Internet Res 2005, 7(4):e48.

49. Mclnnes DK, Saltman DC, Kidd MR: General practitioners' use of computers for prescribing and electronic health records: results from a national survey. Med J Aust 2006, 185(2):88-91.

50. Pharmacy Connectivity Incentive. [http://www.medicareaustralia.gov.au/ provider/pbs/fourth-agreement/pci.shtml]

51. Amsallem E, Kasparian C, Cucherat M, Chabaud S, Haugh M, Boissel JP, Nony P: Evaluation of two evidence-based knowledge transfer interventions for physicians. A cluster randomized controlled factorial design trial: the CardioDAS Study. Fundam Clin Pharmacol 2007, 21(6):631-641.

52. Feldman PH, Murtaugh CM, Pezzin LE, McDonald MV, Peng TR: Just-in-time evidence-based e-mail "reminders" in home health care: impact on patient outcomes. Health Serv Res 2005, 40(3):865-885.

53. Sciamanna CN, Nicholson RA, Lofland JH, Manocchia M, Mui S, Hartman CW: Effects of a Website designed to improve the management of migraines. Headache 2006, 46(1):92-100.

54. Mukohara K, Schwartz MD: Electronic delivery of research summaries for academic generalist doctors: a randomised trial of an educational intervention. Med Educ 2005, 39(4):402-409.

55. Moorjani P, Fortnum H: Dissemination of information to General Practitioners: a questionnaire survey. BMC Fam Pract 2004, 5:27.

56. Sinuff T, Cook D, Giacomini M, Heyland D, Dodek P: Facilitating clinician adherence to guidelines in the intensive care unit: A multicenter, qualitative study. Crit Care Med 2007, 35(9):2083-2089.

57. Bashir K, King M, Ashworth M: Controlled evaluation of brief intervention by general practitioners to reduce chronic use of benzodiazepines. $\mathrm{Br} J$ Gen Pract 1994, 44(386):408-412.

58. Cormack MA, Owens RG, Dewey ME: The effect of minimal interventions by general practitioners on long-term benzodiazepine use. J R Coll Gen Pract 1989, 39(327):408-411.

59. Gorgels WJMJ, Oude Voshaar RC, Mol AJJ, Van De Lisdonk EH, Van Balkom AJLM, Van Den Hoogen HJM, Mulder J, Breteler MHM, Zitman FG: Discontinuation of long-term benzodiazepine use by sending a letter to users in family practice: A prospective controlled intervention study. Drug and Alcohol Dependence 2005, 78(1):49-56. 
60. Morrison JM: Audit and follow-up of chronic benzodiazepine tranquillizer use in one general practice. Family Practice 1990, 7(4):253-257.

61. Zwar NA, Wolk J, Gordon JJ, Sanson-Fisher RW: Benzodiazepine prescribing by GP registrars. A trial of educational outreach. Aust Fam Physician 2000, 29(11):1104-1107.

62. Midlov P, Bondesson A, Eriksson T, Nerbrand C, Hoglund P: Effects of educational outreach visits on prescribing of benzodiazepines and antipsychotic drugs to elderly patients in primary health care in southern Sweden. Family Practice 2006, 23(1):60-64

63. Hagen BF, Armstrong-Esther C, Quail P, Williams RJ, Norton P, Le Navenec CL, Ikuta R, Osis M, Congdon V, Zieb R: Neuroleptic and benzodiazepine use in long-term care in urban and rural Alberta: characteristics and results of an education intervention to ensure appropriate use. Int Psychogeriatr 2005, 17(4):631-652.

64. Avorn J, Soumerai SB, Everitt DE, Ross-Degnan D, Beers MH, Sherman D, Salem-Schatz SR, Fields D: A randomized trial of a program to reduce the use of psychoactive drugs in nursing homes.[see comment]. New England Journal of Medicine 1992, 327(3):168-173.

65. Schmidt I, Claesson CB, Westerholm B, Nilsson LG, Svarstad BL: The impact of regular multidisciplinary team interventions on psychotropic prescribing in Swedish nursing homes. Journal of the American Geriatrics Society 1998, 46(1):77-82.

66. Baker R, Farooqi A, Tait C, Walsh S: Randomised controlled trial of reminders to enhance the impact of audit in general practice on management of patients who use benzodiazepines. Quality in Health Care 1997, 6(1):14-18

67. Holden JD, Hughes IM, Tree A: Benzodiazepine prescribing and withdrawal for 3234 patients in 15 general practices. Family Practice 1994, 11(4):358-362.

68. Pimlott NJG, Hux JE, Wilson LM, Kahan M, Li C, Rosser WW: Educating physicians to reduce benzodiazepine use by elderly patients: A randomized controlled trial. Canadian Medical Association Journal 2003, 168(7):835-839.

69. McClaugherty L: Sedative-Hypnotic Medication Use in 10 Texas Nursing Facilities. The Consultant Pharmacist 1997, 12:149.

70. Gill SS, Misiaszek BC, Brymer C: Improving prescribing in the elderly: a study in the long term care setting. Canadian Journal of Clinical Pharmacology 2001, 8(2):78-83.

71. Crotty M, Whitehead C, Rowett D, Halbert J, Weller D, Finucane P, Esterman A: An outreach intervention to implement evidence based practice in residential care: a randomized controlled trial [ISRCTN67855475]. BMC Health Services Research 2004, 4(1):6.

72. Simon SR, Smith DH, Feldstein AC, Perrin N, Yang $X$, Zhou Y, Platt $R$, Soumerai SB: Computerized prescribing alerts and group academic detailing to reduce the use of potentially inappropriate medications in older people. Journal of the American Geriatrics Society 2006, 54(6):963-968.

73. Monane M, Matthips DM, Nagle BA, Kelly MA: Improving prescribing patterns for the elderly through an online drug utilization review intervention: A system linking the physician, pharmacist, and computer. Journal of the American Medical Association 1998, 280(14):1249-1252.

\section{Pre-publication history}

The pre-publication history for this paper can be accessed here: http://www.biomedcentral.com/1472-6963/10/321/prepub

doi:10.1186/1472-6963-10-321

Cite this article as: Smith and Tett: Improving the use of benzodiazepines-Is it possible? A non-systematic review of interventions tried in the last 20 years. BMC Health Services Research 2010 10:321.

\section{Submit your next manuscript to BioMed Central and take full advantage of:}

- Convenient online submission

- Thorough peer review

- No space constraints or color figure charges

- Immediate publication on acceptance

- Inclusion in PubMed, CAS, Scopus and Google Scholar

- Research which is freely available for redistribution 